\title{
Consumers' Attitudes towards Edible Wild Plants: A Case Study of Noto Peninsula, Ishikawa Prefecture, Japan
}

\author{
Bixia Chen $^{1}$ and Zhenmian Qiu' ${ }^{1,2}$ \\ ${ }^{1}$ Institute of Advanced Studies Operating Unit, United Nations University, Ishikawa/Kanazawa Hirosaka 2-1-1, Kanazawa, \\ Ishikawa 920-0962, Japan \\ ${ }^{2}$ The School of the Economics, Fujian Normal University, Qishan Campus, Minhou County, Fuzhou 350108, China
}

Correspondence should be addressed to Zhenmian Qiu, qiuzhenmian@gmail.com

Received 14 July 2011; Revised 16 November 2011; Accepted 19 December 2011

Academic Editor: Hubert Sterba

Copyright (C 2012 B. Chen and Z. Qiu. This is an open access article distributed under the Creative Commons Attribution License, which permits unrestricted use, distribution, and reproduction in any medium, provided the original work is properly cited.

\begin{abstract}
This study explored the rural revitalizing strategy in FAO's Globally Important Agricultural Heritage System (GIAHS) site in Noto Peninsula, Ishikawa Prefecture of Japan, using a case study of edible wild plants. This study assessed the current and possible future utilization of edible wild plants as one important NTFP by clarifying the attitudes of consumers and exploring the challenges of harvesting edible wild plants. Traditional ecological knowledge associated with edible wild plants and the related attitudes of consumers towards wild plants was documented. A questionnaire survey found that a majority of the respondents held positive attitude towards edible wild plants as being healthy, safe food, part of traditional dietary culture. Increasing demand of edible wild plants from urban residents aroused conflicts with local residents' interest given that around $86 \%$ of the forested hills are private in Noto Region. Non timber forest products (NTFP) extraction can be seen as a tool for creating socioeconomic relationships that are dependent on healthy, biodiverse ecosystems. It was suggested that Japanese Agricultural Cooperatives (JA) and Forestry Cooperatives (FCA) could be involved with GIAHS process. As important traditional dietary and ecological system, edible wild plants should be a part of GIAHS project for rural revitalization.
\end{abstract}

\section{Introduction}

As a hilly and mountainous country, throughout history the people of Japan have developed integrated management knowledge related to forested mountains, in particular, those low hills adjacent to human settlements. Satoyama in Japan has been shaped to a great extent by centuries of management of coppice woodlands on hillside landscapes [1]. A satoyama landscape is an agricultural land use system comprised of secondary forest, farmlands, settlements, and reservoirs [1]. The ecosystems of the secondary woodlands have provided critical services, which benefit human well-being, as well as rich biodiversity [2]. People have lived on the wild plants harvested from the hills and developed rich knowledge related to the uses of wild plants [3]. Some species of wild edible plants, mushrooms, and seaweeds are crucial in various fields of life (e.g., traditional diet system).

Coppice woodlands are managed for the production of wood or charcoal and are cut regularly for this purpose. With the depopulation, migration, and change of life styles after the rapid urbanization in the 1960s, similar to the other parts in the world, however, the forested mountain and traditional knowledge related to it have been degrading [1]. Conversion to coniferous tree plantation to produce timbers has also dramatically changed coppice forest. As a result, the undermanaged forests have threatened the diversity of biology and cultures $[4,5]$. Since the 1980s, the national initiatives have been launched to conserve satoyama landscapes, local and regional initiatives have also increased.

Environmental conservation in satoyama has been the focus of academic and policy-making efforts in Japan recently. Substantial research papers report the concerns of environmental conservation both in Japanese and English [6, 7]. Since the 1980s, laws were stipulated to regulate satoyama landscape mainly by setting limitation to residential and commercial land development. Recent laws relevant to satoyama have been implemented in Japan, including the Act on the Promotion of Nature Restoration (2002), Landscapes Act (2004), and Act on Promotion of Ecotourism (2007) [2]. 


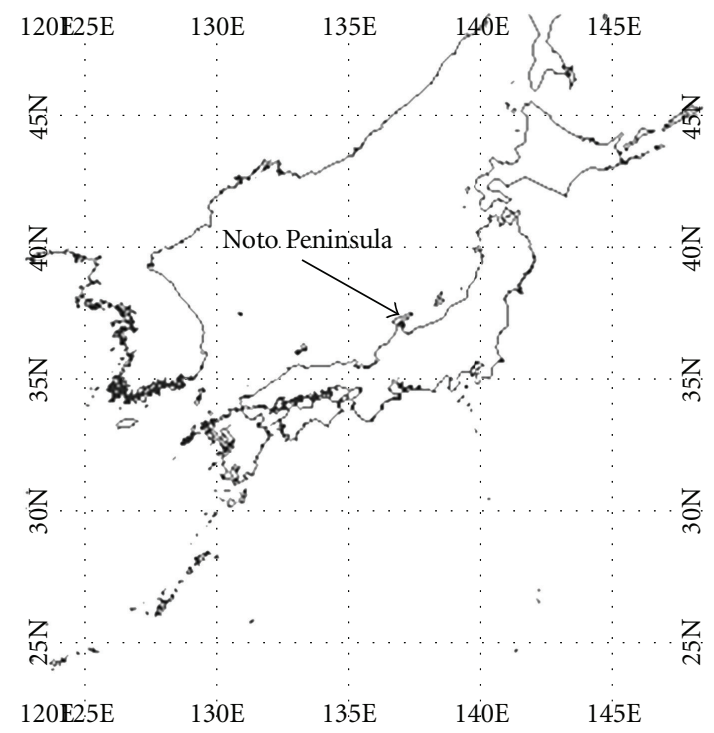

Figure 1: Location of Noto Peninsula

How to conserve the biodiversity in satoyama and revitalize the rural areas has been one of key issues for policy makers at all levels from national government to municipalities. Ecological functions in spite of coppice or timber production become considered as the major purposes of satoyama. In comparison to the substantial efforts of environmental conservation in Japan, uses of natural resources, in particular, non timber forest products (NTFPs) were much less discussed.

The Noto Region in Ishikawa Prefecture (Figure 1), as a microcosm of satoyama, has been designated as a Globally Important Agricultural Heritage Systems (GIAHS) site in June, 2011. GIAHS was defined as "Remarkable land use systems and landscapes which are rich in globally significant biological diversity evolving from the co-adaptation of a community with its environment and its needs and aspirations for sustainable development." [11] As a GIAHS site, it is urgent and vital to develop strategies for dynamic conservation and adaptive management of local agricultural biodiversity, knowledge systems, food and livelihood security, and cultures.

Humans have inhabited the Noto Region for more than one thousand years by utilizing the natural resources both from the hills and the sea. Satoyama in the Noto Region in Japan has abundant species of wild plants which people have utilized for more than one thousand years.

Since 2008, output value from non timber forest product has grown to account for more than half of the gross output from forests in Japan [12]. In contrast to its increasing importance in forestry, studies relevant to wild edible plants are still sporadic. Some early researches mentioned the liking [13] and utilization of edible wild plants [14, 15]. There exists a rising demand of edible wild plants; however, big stores in metropolis were reluctant to sell wild plants due to its small amount of potential customers [16]. Consumers' attitude towards edible wild plants is still little known.
Ishikawa Prefecture can serve as an appropriate survey site for possessing rich plant species and being the center of Hokuriku Area in Japan.

It is expected that results of this study will contribute to the strategies of revitalizing the managed secondary forest ecosystem by promoting recreational use of forest resources. The primary goal of this study is to explore the potential use of natural resources in a sustainable way in the managed secondary forests, which have greatly degraded without thinning in Japan. This study was designed to assess the current and possible future utilization of edible wild plants as one important NTFP by clarifying the attitudes of consumers and exploring the challenges of harvesting edible wild plants. In this study, the effect of demographic characteristic of consumers towards wild plant consumption was also discussed. Sansai is commonly used in Japanese, here it was used to be equivalent to edible wild plants. Sansai also includes various mushrooms besides edible wild plants; however, edible mushrooms are only harvested in autumn, and it needs much related knowledge to tell mushrooms from edible or poisonous. Thus, we only limit sansai to edible wild plants in this study.

Some of the objectives of the study are the documentation of traditional ecological knowledge associated with edible wild plants and the related attitudes of consumers towards wild plants. Specially, the objectives of this study include the following.

(1) Document the species of edible wild plants and the traditional uses of the wild plants, their uses as food or medicine, and their habitat.

(2) Record the traditional knowledge associated with harvest to ensure no harm to natural regeneration.

(3) Survey the attitudes of consumers, including local people, towards edible wild plants. Compare the attitude of residents in remote mountainous areas and urban areas. How demographic characteristics (gender, age, and education) of consumers affect their frequency of purchasing, eating, or harvesting edible wild plants.

(4) Discuss the challenges to the harvest and consumption of edible wild plant. The conflicts of interest of these stakeholders will be discussed and the potential policy implication is also tentatively brought forward.

\section{Survey Sites and Method}

The hilly Noto Peninsula, also usually called the Noto region, is located in the north of Ishikawa Prefecture, which juts out into the Sea of Japan. As a narrow long peninsula extending from the south to the north, the area is characterized by a configuration of closely knit together satoyama and satoumi landscapes, with their associated land uses, diversified livelihoods based on agriculture, forestry and fisheries, lifestyles and customs, and biodiversity. The earliest archaeological evidence of rice cultivation in the region dates back to the mid-Yayoi period (300BC-250AD). 
Two survey sites were selected to interview the respondents, Oozora Farmers' Market and Uchinada Farmers' Market. The former is located in the town of Anamizu in the centre of Noto Peninsula, nearby the entrance to express way. The latter is located in Kanazawa City, the capital city of Ishikawa Prefecture and also the central city in the Hokuriku Region in Japan. These two sites were selected to represent the market in the production place of edible wild plants among the hilly areas and the other one in the urban area as the market center in Ishikawa Prefecture, respectively. These stores are managed as retail store of Agriculture Association Group. They help to sell farmers' product and charge a commission of around $15 \%$ of the total sales.

A combination of qualitative and quantitative data collection methods was used depending on study purposes in this study. The questionnaire survey was conducted in May, 2011, when many species of wild edible plants were collected and sold in the market. During a long vocation at the beginning of May, usually called the Golden Week, warm days in spring have attracted people, not only local residents, but also those from nearby cities to travel. Farmers' market is among one of best popular sites for visitors. Visitors in this season can represent a wide variety besides local residents.

In depth interviews were conducted with multistakeholders relevant to the utilization of wild edible plants: collectors, consumers, and specialists of wild edible plants, forest owners, Forest Cooperation Association in Noto, and municipalities. Interviews lasted around an hour at average. We communicated in Japanese and notes were also taken in Japanese. Then interviews were translated into English. Consumers were briefly interviewed after a questionnaire sheet completed when time allowed to grasp additional consumers' attitudes toward wild edible plants.

A list of edible wild plants was compiled after consulting with local experts of vegetation. First, we obtained a list of edible wild plants from the Ishikawa Prefecture office. The list was revised and extensively recompiled by deleting extinct species and wrong data and adding missing data. In order to identify plant species and habitat and obtain relevant ecological knowledge, we have visited the forest to pick up edible wild plants with Ms. Fujiko Taniguchi, who is a registered edible plant cooking adviser with vegetation identification experience of around $50 \mathrm{yrs}$., to collect edible plants during the three months of April-June, 2011. We also have spent several months to collect basic ecological knowledge by learning cooking and preservation skills of edible wild plants. Ecological knowledge of plant habitats and different ways of use were documented in detail.

A questionnaire survey was designed to clarify the species and frequency of wild edible plants have been widely collected, purchased or eaten. The respondents were split into three groups according to their residence, residents in Noto Region (also refer to group I in this paper), in Ishikawa Prefecture except Noto Region (group II), and outside Ishikawa Prefecture (group III).

The questionnaire survey is comprised of four different sections. The first section contained the personal profile of the respondents, including questions related to ages, education, and their residence. The second section includes the frequency and items of edible plants that they harvest, purchase, or eat. The third section includes questions of their attitudes toward edible wild plants. Respondents were asked to comment on their perceived factors of being delicious, healthy, cheap at price, easy to buy, safe food without the use of pesticides, environmental friendly without the input of chemical fertilizers. A five-point Likert scale ranging from "strongly agree $(=5)$, agree $(=4)$, no idea $(=3)$, disagree $(=2)$, highly disagree $(=1)$ " was used to get the respondent's opinion on edible wild plants. In the last section of the open question, the respondent was asked to freely write down their opinions related to harvest, purchase, and cooking of edible wild plants.

A total number of 208 copies of questionnaires were collected and completed without missing values in the two survey sites: 106 copies in Anamizu Farmers' Market and 102 copies in Uchinada Farmers' Market. About 43 respondents in Anamizu and 35 in Uchinada refused to answer the questionnaire. Each copy was checked and the respondent was asked to complete if any missing values found. All completed questionnaire sheets collected from the two survey sites were split into three groups by respondents' residence, Noto Region (group I), Ishikawa Prefecture other than Noto Region (group II), outside Ishikawa Prefecture (group III). In this study, we adopted the definition of Noto Region according to GIAHS site project as four cities and four towns in the Noto Peninsula on the Japan Sea side of Japan's main island, Honshu: Nanao City, Wajima City, Suzu City, Hakui City, Shika Town, Nakanoto Town, Anamizu Town, and Noto Town. The respondents of group II can be described as urban residents who are majorly from Kanazawa and its neighboring areas to the south of Noto Region. Group III consists of residents from metropolis of Tokyo, Kyoto, and Osaka and other mountainous areas.

The descriptive profile of respondents is listed in Table 1. Since the number of the results of group III is much less than groups I and II, this study will be discussed based on the comparison between groups I and II, while using group III as reference, moreover, respondents of group III are a mix of rural and urban residents. The results had a good balance of male and female respondents with female respondents being slightly more than male ones. Respondents from group I are a little older than group II, which is consistent with the current population situation with the increasing ageing people in the rural areas.

The collected data were further calculated to analyze whether the factors of respondents' profile: the age, city of residence, education, and even different survey sites affected the frequency that they bought, harvested, or ate edible wild plants. Two categories of respondents' behavior were classified: high frequency referred to those who purchased, harvested, or ate more than once a week; low frequency referred to those who never purchased, harvested, or ate wild edible plants or only a few times a year. Because these two categories were unordered, binary logistic model were used for analysis. SPSS 16.0 was used to estimate the binary logistic model. 
TABLE 1: A full list of wild plants used as food and medicine.

\begin{tabular}{|c|c|c|c|c|}
\hline Species & Family & Plant parts used for food & Plant parts used for medicine & Habitat \\
\hline $\begin{array}{l}\text { Acanthopanax } \\
\text { sciadophylloides }\end{array}$ & Eleutherococcus & Sprout & & Rice paddy, wetland \\
\hline Actinidia arguta & Actinidiaceae & Fruit & Gall & Mountain \\
\hline Actinidia polygama & Actinidiaceae & Seed & & Forest fringe, mountain forest \\
\hline $\begin{array}{l}\text { Adenophora } \\
\text { remotiflora }\end{array}$ & Campanulaceae & Sprout & & $\begin{array}{l}\text { Hill and mountain, forest fringe, } \\
\text { Valley, bank }\end{array}$ \\
\hline $\begin{array}{l}\text { Adenophora } \\
\text { triphylla }\end{array}$ & Campanulaceae & Young leaf & & Hill and mountain \\
\hline $\begin{array}{l}\text { Aesculus } \\
\text { hippocastanum }\end{array}$ & Hippocastanaceae & Seed & The whole grass & Open space, road side \\
\hline Akebia quinata & Akebia & Sprout, vine, fruit & & Coast, bank, mountain \\
\hline Allium grayi & Liliaceae & Young leaf, root & Leaf & $\begin{array}{l}\text { Wetland, stone fence, rock, } \\
\text { garden }\end{array}$ \\
\hline $\begin{array}{l}\text { Allium } \\
\text { schoenoprasum }\end{array}$ & Liliaceae & Scaly root-stalk, Young leaf & & Coast, mountain, bank side \\
\hline $\begin{array}{l}\text { Allium victorialis } \\
\text { var. platyphyllum }\end{array}$ & Liliaceae & Young leaf & Root, stem & Valley, nearby the spring \\
\hline $\begin{array}{l}\text { Amana edulis } \\
\text { (Miq.) Honda }\end{array}$ & Liliaceae & Scaly root-stalk, Sprout & & Grassland \\
\hline Amaranthus lividus & Amaranthaceae & Sprout & & River bank, roadside, coast \\
\hline $\begin{array}{l}\text { Amaranthus lividus } \\
\text { Loisel. }\end{array}$ & Amaranthaceae & $\begin{array}{l}\text { Young leaf, leaf, Top of the } \\
\text { stem }\end{array}$ & & Forest, grassland \\
\hline Anemone flaccida & Ranunculaceae & Sprout, flower & Root & Grassland \\
\hline Angelica pubescens & Apiaceae & Sprout & Root, the whole Plant & Road side, open field \\
\hline Anthriscus sylvestris & Apiaceae & Sprout, young Leaf & & Coppice, beech forest \\
\hline Apios fortunei & Fabaceae & Tuberous root & The whole grass & $\begin{array}{l}\text { Sunny wetland, abandoned field, } \\
\text { at the foot of a mountain }\end{array}$ \\
\hline Aralia cordata & Eleutherococcus & Sprout & The whole grass & $\begin{array}{l}\text { Field, road side, grassland, } \\
\text { garden }\end{array}$ \\
\hline Aralia elata & Eleutherococcus & Sprout & Bulb, flower & Hill and mountain, coast \\
\hline Artemisia princeps & Asteraceae & Young leaf & & Hill and mountain \\
\hline $\begin{array}{l}\text { Aruncus dioicus } \\
\text { var. tenuifolius }\end{array}$ & Rosaceae & Sprout & & Open field, road side \\
\hline $\begin{array}{l}\text { Asparagus } \\
\text { schoberioides }\end{array}$ & Liliaceae & Sprout & & $\begin{array}{l}\text { Forest in the mountain, forest } \\
\text { fringe }\end{array}$ \\
\hline $\begin{array}{l}\text { Aster glehni var. } \\
\text { hondoensis }\end{array}$ & Asteraceae & Young leaf & & $\begin{array}{l}\text { Forest fringe, mountain road } \\
\text { side, grassland }\end{array}$ \\
\hline Aster leiophyllus & Asteraceae & Sprout & & $\begin{array}{l}\text { Mountain forest, wetland in the } \\
\text { plain }\end{array}$ \\
\hline Aster scaber & Asteraceae & Sprout & & Coppice, mountain forest \\
\hline Aster yomena & Asteraceae & Young leaf & & Coast, hill and mountain, field \\
\hline $\begin{array}{l}\text { Astilbe thunbergii } \\
\text { var. congesta }\end{array}$ & Saxifragaceae & Sprout & & Mountain forest \\
\hline $\begin{array}{l}\text { Atractylodes } \\
\text { japonica }\end{array}$ & Asteraceae & Sprout & Pericarp & Hill and mountain, bush \\
\hline Begonia grandis & Begoniaceae & Flower & Root stem, root, leaf & Bank of field \\
\hline $\begin{array}{l}\text { Benthamidia } \\
\text { japonica }\end{array}$ & Cornaceae & Flower & Fatty oil of seed & Coast, low hill \\
\hline Brasenia schreberi & Nymphaeaceae & Sprout & & Mountain forest \\
\hline $\begin{array}{l}\text { Broussonetia } \\
\text { kazinoki }\end{array}$ & Moraceae & Fruit & & Forest mountain \\
\hline Buckleya lanceolata & Santalaceae & Young seed & & Coast, sandy land \\
\hline Calystegia japonica & Convolvulaceae & $\begin{array}{l}\text { Young leaf, underground } \\
\text { stem, top of the vine }\end{array}$ & & Coast, sandy land \\
\hline
\end{tabular}


TABle 1: Continued.

\begin{tabular}{|c|c|c|c|c|}
\hline Species & Family & Plant parts used for food & Plant parts used for medicine & Habitat \\
\hline $\begin{array}{l}\text { Calystegia } \\
\text { soldanella (L.) } \\
\text { Roem. et Schult. }\end{array}$ & Convolvulaceae & Flower & & Forest, grassland \\
\hline Camellia japonica & Theaceae & Flower, seed & & River bank, forest fringe \\
\hline Camellia sinensis & Theaceae & Sprout, flower & & Sandy coast \\
\hline $\begin{array}{l}\text { Campanula } \\
\text { punctata Lam. }\end{array}$ & Campanulaceae & Sprout & & Forest \\
\hline Caprifoliaceae & Fagaceae & Fruit & & Abandoned land in satoyama \\
\hline $\begin{array}{l}\text { Capsella } \\
\text { bursa-pastoris }\end{array}$ & Brassicaceae & Young sprout & Root stem & Road side, plain, bank, open field \\
\hline Cardamine flexuosa & Brassicaceae & Sprout, young leaf & & Hill and mountain \\
\hline $\begin{array}{l}\text { Cardamine } \\
\text { leucantha }\end{array}$ & Brassicaceae & Leaf, stem & Seed & Street side, park, temple \\
\hline $\begin{array}{l}\text { Cardiocrinum } \\
\text { cordatum }\end{array}$ & Liliaceae & $\begin{array}{l}\text { Scaly root-stalk, young leaf, } \\
\text { stem }\end{array}$ & Scaly root-stalk & $\begin{array}{l}\text { Roadside in the mountain, } \\
\text { somber forest }\end{array}$ \\
\hline Castanea crenata & Fagaceae & Seed & & Forest \\
\hline Castanea crenata & Fagaceae & Seed & & Grassland, forest fringe \\
\hline Cayratia japonica & Vitaceae & Sprout & & $\begin{array}{l}\text { Open field, road side, abandoned } \\
\text { land, bank }\end{array}$ \\
\hline $\begin{array}{l}\text { Chenopodium } \\
\text { album var. } \\
\text { centrorubrum }\end{array}$ & Chenopodiaceae & Sprout & Leaf, stem, utricle & Field, road side \\
\hline $\begin{array}{l}\text { Circium } \\
\text { nipponicum }\end{array}$ & Asteraceae & Sprout, stem, root & & Forest, forest fringe, grassland \\
\hline Cirsium yezoense & Asteraceae & Sprout, young stem, root & & Grassland, road side, riverside \\
\hline $\begin{array}{l}\text { Clerodendron } \\
\text { trichotomum }\end{array}$ & Verbenaceae & Young leaf & $\begin{array}{l}\text { Leaf, phyllary, astringent } \\
\text { skin }\end{array}$ & Coppice, hill and mountain \\
\hline Clethra barbinervis & Clethraceae & Sprout & & Flatland, coppice \\
\hline $\begin{array}{l}\text { Codonopsis } \\
\text { lanceolata }\end{array}$ & Campanulaceae & Sprout & Fruit, root bark, leaf & Open field, bank, forest, \\
\hline $\begin{array}{l}\text { Commelina } \\
\text { communis }\end{array}$ & Commelinaceae & Young leaf, stem & Root & Grassland, forest, forest fringe \\
\hline $\begin{array}{l}\text { Corylus sieboldiana } \\
\text { Blume }\end{array}$ & Betulaceae & & Endodermis & Bright forest \\
\hline $\begin{array}{l}\text { Crassocephalum } \\
\text { crepidioides }\end{array}$ & Asteraceae & Young leaf & Parts above the ground & Hill and mountain \\
\hline $\begin{array}{l}\text { Cuculus } \\
\text { poliocephalus }\end{array}$ & Liliaceae & Young sprout & & Forest fringe, grassland \\
\hline $\begin{array}{l}\text { Cymbidium } \\
\text { goeringii }\end{array}$ & Orchidaceae & Flower, stem & & Swamp, filed \\
\hline Dioscorea japonica & Dioscoreaceae & Root & & High mountain, forest fringe \\
\hline $\begin{array}{l}\text { Diospyros kaki var. } \\
\text { sylvestris Makino }\end{array}$ & Ebenaceae & Fruit, leaf & & Mountain, plain, Forest \\
\hline $\begin{array}{l}\text { Diplazium } \\
\text { squamigerum }\end{array}$ & Woodsiaceae & Sprout & & Sunny hillside \\
\hline $\begin{array}{l}\text { Elaeagnus } \\
\text { umbellata }\end{array}$ & Elaeagnaceae & Fruit & & $\begin{array}{l}\text { Riverside and roadside in the } \\
\text { mountain }\end{array}$ \\
\hline $\begin{array}{l}\text { Elatostema } \\
\text { laetevirens }\end{array}$ & Urticaceae & Leaf, stem & Leaf, fruit & Hill and mountain, forest fringe \\
\hline $\begin{array}{l}\text { Elatostema } \\
\text { umbellatum var. } \\
\text { majus }\end{array}$ & Urticaceae & Sprout, stem, seed & The whole grass, seed & Road side, open space \\
\hline $\begin{array}{l}\text { Eleutherococcus } \\
\text { Maxim. }\end{array}$ & Eleutherococcus & Sprout & & $\begin{array}{l}\text { Wetland, mountain forest, forest } \\
\text { fringe }\end{array}$ \\
\hline
\end{tabular}


TABle 1: Continued.

\begin{tabular}{|c|c|c|c|c|}
\hline Species & Family & Plant parts used for food & Plant parts used for medicine & Habitat \\
\hline $\begin{array}{l}\text { Epimedium } \\
\text { grandiflorum }\end{array}$ & Berberidaceae & Young leaf & Root & Open field \\
\hline Equisetum arvense & Equisetaceae & Bud & The whole grass & $\begin{array}{l}\text { Wet road side, open space, north } \\
\text { of the house }\end{array}$ \\
\hline Equisetum arvense & Equisetaceae & The whole plant & & Forest, riverside \\
\hline Erigeron annuus & Asteraceae & Young leaf, stem & & Hill and mountain, forest fringe \\
\hline $\begin{array}{l}\text { Erigeron } \\
\text { philadelphicus }\end{array}$ & Asteraceae & & Root, flower & Hill and mountain, sunny place \\
\hline $\begin{array}{l}\text { Euonymus } \\
\text { sieboldianus }\end{array}$ & Celastraceae & Sprout & & Open field, forest fringe \\
\hline Eutrema japonica & Brassicaceae & Root, flower, stem & Underground stem & Mountain forest \\
\hline Eutrema tenuis & Brassicaceae & Leaf, stem, flower & & Grassland \\
\hline $\begin{array}{l}\text { Fagopyrum } \\
\text { cymosum }\end{array}$ & Polygonaceae & Above ground sprout & & Open field, bank \\
\hline Fagus crenata & Fagaceae & Sprout, seed & & Mountain \\
\hline $\begin{array}{l}\text { Farfugium } \\
\text { japonicum }\end{array}$ & Asteraceae & Bud, sprout & & Fallow field, wetland \\
\hline Gamblea innovans & Eleutherococcus & Sprout & & Sunny hillside \\
\hline $\begin{array}{l}\text { Geranium } \\
\text { thunbergii }\end{array}$ & Geraniaceae & Young leaf & & Pond \\
\hline $\begin{array}{l}\text { Gesneriaceae } \\
\text { Dumortier nom. } \\
\text { cons. }\end{array}$ & Gesneriaceae & Leaf & & Sandy coast \\
\hline Ginkgo biloba & Ginkgoaceae & Seed & & Coppice, beech forest \\
\hline $\begin{array}{l}\text { Glechoma } \\
\text { hederacea }\end{array}$ & Lamiaceae & Sprout, stem & Leaf, bud & $\begin{array}{l}\text { Grassland, open space, forest } \\
\text { fringe }\end{array}$ \\
\hline Glehnia littoralis & Apiaceae & Sprout & & Coppice, beech forest \\
\hline Gnaphalium affine & Asteraceae & Sprout & & Paddy field, fallow field \\
\hline $\begin{array}{l}\text { Helianthus } \\
\text { tuberosus }\end{array}$ & Asteraceae & Tuberous root & & $\begin{array}{l}\text { Wetland in the mountain, } \\
\text { riverside, fallow land }\end{array}$ \\
\hline Helwingia japonica & Cornaceae & Sprout, fruit & Seed & Abandoned land, mountain \\
\hline $\begin{array}{l}\text { Hemerocallis fulva } \\
\text { var. kwanso }\end{array}$ & Liliaceae & Young leaf, bud, flower & Root, stem & $\begin{array}{l}\text { Hill and mountain, grassland, } \\
\text { garden }\end{array}$ \\
\hline $\begin{array}{l}\text { Hosta } \\
\text { albo-marginata }\end{array}$ & Liliaceae & Sprout, leaf stalk & $\begin{array}{l}\text { Leaf, trichome, and fimbrae } \\
\text { on the leaf }\end{array}$ & Open field, roadside \\
\hline Hosta montana & Liliaceae & Sprout, leaf stalk & & $\begin{array}{l}\text { Wet place in the mountain, } \\
\text { cultivated nearby settlement }\end{array}$ \\
\hline Houttuynia cordata & Saururaceae & Sprout, root & Root stem & Wetland in the mountain \\
\hline Hovenia dulcis & Rhamnaceae & Fruit & & Garden \\
\hline $\begin{array}{l}\text { Hydrangea } \\
\text { petiolaris }\end{array}$ & Hydrangeaceae & Young leaf & Stem, root & Mountain forest \\
\hline $\begin{array}{l}\text { Imperata cylindrica } \\
\text { L. }\end{array}$ & Poaceae & Flower & & Mountain forest \\
\hline $\begin{array}{l}\text { Juglans } \\
\text { mandshurica var. } \\
\text { sachalinensis }\end{array}$ & Juglandaceae & Seed & $\begin{array}{l}\text { Parts above ground, root } \\
\text { stem }\end{array}$ & Field, nearby human settlement \\
\hline $\begin{array}{l}\text { Kalimeris } \\
\text { pinnatifida }\end{array}$ & Asteraceae & Sprout & Leaf, bark & Hill and mountain \\
\hline $\begin{array}{l}\text { Kalopanax } \\
\text { septemlobus }\end{array}$ & Eleutherococcus & Sprout & & Grassland, forest fringe \\
\hline $\begin{array}{l}\text { Laportea } \\
\text { macrostachya }\end{array}$ & Urticaceae & Sprout, flower & & $\begin{array}{l}\text { Mountain forest, wetland on the } \\
\text { cliff }\end{array}$ \\
\hline $\begin{array}{l}\text { Lapsana } \\
\text { apogonoides }\end{array}$ & Asteraceae & Young leaf & & Mountain forest, riverside \\
\hline
\end{tabular}


Table 1: Continued.

\begin{tabular}{|c|c|c|c|c|}
\hline Species & Family & Plant parts used for food & Plant parts used for medicine & Habitat \\
\hline Lathyrus japonicus & Fabaceae & & & Mountain, garden \\
\hline Lilium auratum & Liliaceae & Root & & Forest fringe, mountain \\
\hline $\begin{array}{l}\text { Lilium } \\
\text { lancifoliumThunb. }\end{array}$ & Liliaceae & Scaly root-stalk & Scaly root-stalk & \\
\hline $\begin{array}{l}\text { Lilium leichtlinii } \\
\text { var. tigrinum }\end{array}$ & Liliaceae & Root & & Forest \\
\hline Lonicera japonica & Caprifoliaceae & Sprout & & Edge of the mountain \\
\hline $\begin{array}{l}\text { Lycium } \\
\text { rhombifolium }\end{array}$ & Solanaceae & Young leaf, fruit & & Coppice, low hill \\
\hline Lythrum anceps & Lythraceae & & & Forest \\
\hline Magnolia kobus & Magnoliaceae & Flower, seed & & Riverside, wetland in the forest \\
\hline Magnolia obovata & Magnoliaceae & Young leaf, fallen leaf & & Forest \\
\hline Malus toringo & Rosaceae & Fruit & Seed & Cultivated in a swamp or river \\
\hline $\begin{array}{l}\text { Matteuccia } \\
\text { struthiopteris }\end{array}$ & Dryopteridaceae & Sprout & & $\begin{array}{l}\text { Open field, bank, road side, } \\
\text { plateau }\end{array}$ \\
\hline Metaplexis japonica & Asclepiadaceae & & & Abandoned land, bank, field \\
\hline Moraceae bombycis & Moraceae & Young leaf, fruit & & Swamp \\
\hline $\begin{array}{l}\text { Nasturtium } \\
\text { officinale }\end{array}$ & Brassicaceae & $\begin{array}{l}\text { Sprout, young stem, young } \\
\text { leaf }\end{array}$ & & $\begin{array}{l}\text { Road side field, grass land, ridge } \\
\text { between rice fields }\end{array}$ \\
\hline Nelumbo nucifera & Nelumbonaceae & Root, fruit, receptacle & & Forest, riverside \\
\hline Oenanthe javanica & Apiaceae & Young leaf & & Paddy field, wetland \\
\hline Osmunda japonica & Osmundaceae & Sprout & Root stem & $\begin{array}{l}\text { Riverside, open field, sandy land, } \\
\text { hill, and mountain }\end{array}$ \\
\hline Padus grayana & Rosaceae & $\begin{array}{l}\text { Young flower, young fruit, } \\
\text { red fruit }\end{array}$ & & Sandy coast, rock \\
\hline $\begin{array}{l}\text { Parasenecio } \\
\text { delphiniifolius }\end{array}$ & Asteraceae & Sprout, stem & Root, bark, stem, fruit & Forest fringe, field, mountain \\
\hline Patrinia villosa & Valerianaceae & Young leaf & & Hill and mountain \\
\hline Petasites japonicus & Asteraceae & Flower, leaf stalk, leaf & Vine fruit & Forest, park \\
\hline $\begin{array}{l}\text { Phellodendron } \\
\text { amurense }\end{array}$ & Rutaceae & & Root stem, root leaf & Abandoned land, bush, roadside \\
\hline $\begin{array}{l}\text { Phyllostachys } \\
\text { heterocyclaf. } \\
\text { pubescens }\end{array}$ & Poaceae & Root, sprout, balk & & Coppice \\
\hline $\begin{array}{l}\text { Phyllostachys nigra } \\
\text { var. henonis }\end{array}$ & Poaceae & Above ground sprout & Root stem & Hill slope, bank \\
\hline $\begin{array}{l}\text { Phytolacca } \\
\text { esculenta } V\end{array}$ & Phytolaccaceae & Root, leaf & & Forest \\
\hline $\begin{array}{l}\text { Picris hieracioides } \\
\text { subsp. japonica }\end{array}$ & Asteraceae & Young leaf & & $\begin{array}{l}\text { Wetland in the mountain, } \\
\text { riverside, fallow land }\end{array}$ \\
\hline Plantago asiatica & Plantaginaceae & Young leaf & & Sunny grassland, road side \\
\hline $\begin{array}{l}\text { Platycodon } \\
\text { grandiflorus }\end{array}$ & Campanulaceae & & & $\begin{array}{l}\text { River inside the remote } \\
\text { mountain, forest }\end{array}$ \\
\hline $\begin{array}{l}\text { Polygonatum } \\
\text { odoratum }\end{array}$ & Liliaceae & Sprout & & Forest fringe, grassland \\
\hline $\begin{array}{l}\text { Polystichum } \\
\text { triperon }\end{array}$ & Polypodiaceae & Sprout & Seed & Mountain forest, shrine \\
\hline $\begin{array}{l}\text { Polygonatum } \\
\text { falcatum }\end{array}$ & Fabaceae & Sprout & & Forest \\
\hline $\begin{array}{l}\text { Polygonum } \\
\text { hydropiper L. }\end{array}$ & Polygonaceae & Young leaf & & Forest, open field, coppice \\
\hline $\begin{array}{l}\text { Polygonum } \\
\text { thunbergii }\end{array}$ & Polygonaceae & Sprout, young leaf & Young leaf & Field, coppice, an afforested area \\
\hline Portulaca oleracea & Portulacaceae & Leaf, stem & & Wetland, wet plain \\
\hline
\end{tabular}


Table 1: Continued.

\begin{tabular}{|c|c|c|c|c|}
\hline Species & Family & Plant parts used for food & Plant parts used for medicine & Habitat \\
\hline $\begin{array}{l}\text { Prunella vulgaris } \\
\text { subsp. Asiatica }\end{array}$ & Lamiaceae & & & Grassland, forest fringe \\
\hline $\begin{array}{l}\text { Pteridium } \\
\text { aquilinum }\end{array}$ & Dennstaedtiaceae & Sprout, stem & & Coppice, deciduous forest \\
\hline Pueraria lobata & Fabaceae & $\begin{array}{l}\text { Flower, vine sprout, root, } \\
\text { bud }\end{array}$ & & Forest fridge in the warm place \\
\hline Raphanus sativus & Brassicaceae & Sprout, root, fruit & Stem, leaf & Hill and mountain \\
\hline $\begin{array}{l}\text { Reynoutria } \\
\text { japonica }\end{array}$ & Polygonaceae & Sprout & & Coppice \\
\hline $\begin{array}{l}\text { Rhododendron } \\
\text { kaempferi }\end{array}$ & Ericaceae & Flower & & Forest fringe, coppice \\
\hline $\begin{array}{l}\text { Robinia } \\
\text { pseudo-acacia }\end{array}$ & Fabaceae & Sprout, flower & & Forest floor, riverside \\
\hline Rorippa indica & Brassicaceae & Sprout & & Field \\
\hline Rosa rugosa & Rosaceae & Fruit & & Grassland, bank, field \\
\hline Rubus crataegifolius & Rosaceae & Fruit & & Cultivated in satoyama \\
\hline Rubus hirsutus & Rosaceae & Fruit & & Forest fringe, riverside \\
\hline $\begin{array}{l}\text { Rubus palmatus } \\
\text { var. coptophyllus }\end{array}$ & Rosaceae & Fruit & The whole grass & Sunny roadside, field, garden \\
\hline Rubus parvifolius & Liliaceae & Fruit & & $\begin{array}{l}\text { Valley, wetland in the mountain, } \\
\text { shady forest }\end{array}$ \\
\hline Rumex acetosa & Polygonaceae & Sprout & & Wetland, ridge of field \\
\hline Salsola komarovii & Chenopodiaceae & Sprout, leaf & & Field, open space \\
\hline $\begin{array}{l}\text { Sambucus } \\
\text { sieboldiana }\end{array}$ & Caprifoliaceae & Sprout & Parts above ground & Open field, plain \\
\hline $\begin{array}{l}\text { Sanguisorba } \\
\text { officinalis } L \text {. }\end{array}$ & Rosaceae & Sprout & Stem, leaf & $\begin{array}{l}\text { Sunny side of the hill and } \\
\text { mountain }\end{array}$ \\
\hline Sasa palmata & Poaceae & Young leaf & Bulb & $\begin{array}{l}\text { Somber forest, fringe of the } \\
\text { mountain road }\end{array}$ \\
\hline $\begin{array}{l}\text { Saxifraga fortunei } \\
\text { var. incisolobata }\end{array}$ & Saxifragaceae & Leaf, stem & & Coast, forest, forest fringe \\
\hline $\begin{array}{l}\text { Saxifraga } \\
\text { stolonifera }\end{array}$ & Saxifragaceae & Leaf, stem & & Mountain forest, bush \\
\hline $\begin{array}{l}\text { Schisandra } \\
\text { nigra/Schisandra } \\
\text { repanda }\end{array}$ & Schisandraceae & Fruit & Vine & Mountain \\
\hline Senecia pierotii & Asteraceae & Sprout, young stem, flower & & Riverside \\
\hline $\begin{array}{l}\text { Senecio } \\
\text { cannabifolius }\end{array}$ & Asteraceae & Sprout & & Sunny grassland, bank \\
\hline Smilax china & Smilacaceae & Sprout, leaf, fruit & & Grassland, forest fringe \\
\hline Smilax riparia & Liliaceae & Sprout & & $\begin{array}{l}\text { Grassland, road side, open space, } \\
\text { field }\end{array}$ \\
\hline Sonchus oleraceus & Asteraceae & Sprout & & Rocky coast \\
\hline Sorbus commixta & Rosaceae & Fruit & Parts above ground & Open field, field \\
\hline Staphylea bumalda & Staphyleaceae & Sprout, flower & & Forest \\
\hline $\begin{array}{l}\text { Stauntonia } \\
\text { hexaphylla }\end{array}$ & Akebia & Fruit, sprout & Vine in autumn & Mountain, garden \\
\hline Stellaria aquaticum & Caryophyllaceae & Young leaf, the whole grass & & Forest fringe, roadside \\
\hline $\begin{array}{l}\text { Stellaria media (L.) } \\
\text { Villars }\end{array}$ & Caryophyllaceae & & & Forest \\
\hline Stellaria neglecta & Caryophyllaceae & & & Forest \\
\hline Syneilesis palmata & Asteraceae & Sprout, stem & Peel of fruit, seed & $\begin{array}{l}\text { Fringe of a swamp in the } \\
\text { mountain }\end{array}$ \\
\hline
\end{tabular}


TABle 1: Continued.

\begin{tabular}{|c|c|c|c|c|}
\hline Species & Family & Plant parts used for food & Plant parts used for medicine & Habitat \\
\hline Synurus pungens & Asteraceae & Sprout & & $\begin{array}{l}\text { Forest on the hill and mountain, } \\
\text { edge of the forest }\end{array}$ \\
\hline $\begin{array}{l}\text { Taraxacum } \\
\text { officinale }\end{array}$ & Asteraceae & Leaf, stem & & Coppice, beech forest \\
\hline $\begin{array}{l}\text { Tetragonia } \\
\text { tetragonioides }\end{array}$ & Tetragoniaceae & Young leaf & Stem, leaf, root stem & Shady place nearby the sea \\
\hline Torreya nucifera & Taxaceae & Seed & Leaf & Cliff inside the mountain \\
\hline Trapa japonica & Trapaceae & Fruit & Flower, fruit & Coast, sandy land \\
\hline $\begin{array}{l}\text { Vaccinium } \\
\text { oldhamii }\end{array}$ & Ericaceae & Fruit & The whole grass & Abandoned land, road side, field \\
\hline $\begin{array}{l}\text { Vaccinium hirtum } \\
\text { var. pubecens }\end{array}$ & Ericaceae & Fruit & Root & Sunny side of hill and mountain \\
\hline $\begin{array}{l}\text { Viburnum } \\
\text { dilatatum }\end{array}$ & Caprifoliaceae & Fruit & & Forest floor \\
\hline $\begin{array}{l}\text { Viburnum } \\
\text { furcatum }\end{array}$ & Caprifoliaceae & Fruit & & Forest, park \\
\hline Vicia sepium & Fabaceae & $\begin{array}{l}\text { Young leaf, flower, young } \\
\text { fruit }\end{array}$ & & Cultivated in satoyama, forest \\
\hline Vicia unijuga & Fabaceae & Young leaf & & Satoyama forest, field \\
\hline $\begin{array}{l}\text { Vigna angularis } \\
\text { var. nipponensis }\end{array}$ & Fabaceae & seed & & Wetland, dike \\
\hline Viola mandshurica & Violaceae & Flower, leaf & & Rocky side of swamp \\
\hline Viola vaginata & Violaceae & Flower, root, young leaf & & Forest fringe, grassland \\
\hline Vitis coignetiae & Vitaceae & Sprout, fruit & & Open field \\
\hline Vitis ficifolia & Vitaceae & Fruit, sprout, young leaf & & Forest fringe, open field \\
\hline Vitis flexuosa & Vitaceae & Fruit, sprout, young leaf & Bud & Hill, flat land, forest, field \\
\hline Wisteria floribunda & Fabaceae & Flower, young leaf, seed & & Wetland, riverside \\
\hline $\begin{array}{l}\text { Zanthoxylum } \\
\text { piperitum }\end{array}$ & Rutaceae & $\begin{array}{l}\text { Young fruit, young leaf, } \\
\text { ripened fruit }\end{array}$ & & Ridge of fields \\
\hline $\begin{array}{l}\text { Zanthoxylum } \\
\text { piperitum (L.) DC }\end{array}$ & Rutaceae & & & Riverside, wetland in the forest \\
\hline Zingiber mioga & Zingiberaceae & Sprout, flower, stem & The whole plant & Grassland, field, garden \\
\hline
\end{tabular}

TABLE 2: The number of species of wild plants used as food and medicine.

\begin{tabular}{lccc}
\hline & Total & Food & Medicine \\
\hline $\begin{array}{l}\text { Number } \\
\text { Family } \\
\text { Species }\end{array}$ & 67 & 65 & 60 \\
$\begin{array}{l}\text { Parts to be } \\
\text { used }\end{array}$ & 184 & 176 & Sprout, flowers, soft stem, root, seed, bark, and fruit \\
Habitat & & Open field, the border of the forest, rivers, paddy fields, forest, and shoreline
\end{tabular}

Note: this table was summarized under the instruction of Ms. Fujiko Tanikuchi, with a reference to the books as follows: Flora in Kaga and Noto [8]; Best Guide to Wild Edible Plants [9]; Dictionary of Trees and Herbs Used as Medicine [10].

\section{Results and Discussion}

3.1. Documentation of Use of Edible Wild Plants. The edible wild plants were compiled with the help of a local vegetation specialist named Fujiko Taniguchi and the reference to some flora books [8-10]. The detail of their habitat, uses as food or medicine, and which part of the plant could be used were tallied. We got a result of around 184 species, belonging to 67 families, which can be used as food both/or medicine in Noto Region (Table 2). Among them, around 176 species belonging to 65 families are edible; around 60 species belonging to 36 families can be used as medicine (Table 3 ). Almost all parts of the plants can be used, including the sprouts, flowers, fruits, seeds, soft stems, and roots. 
TABle 3: Profile of respondents.

\begin{tabular}{|c|c|c|c|c|c|c|c|c|}
\hline \multirow[b]{2}{*}{ Gender } & \multicolumn{2}{|c|}{ Total } & \multicolumn{2}{|c|}{ Noto Peninsula (group I) } & \multicolumn{2}{|c|}{$\begin{array}{l}\text { Ishikawa Prefecture } \\
\text { except Noto (group } \\
\text { II) }\end{array}$} & \multicolumn{2}{|c|}{$\begin{array}{l}\text { Outside Ishikawa } \\
\text { Prefecture (group III) }\end{array}$} \\
\hline & Number & Frequency & Number & Frequency & Number & Frequency & Number & Frequency \\
\hline Male & 93 & $44.7 \%$ & 32 & $42.1 \%$ & 47 & $43.5 \%$ & 14 & $58.3 \%$ \\
\hline Female & 115 & $55.3 \%$ & 44 & $57.9 \%$ & 61 & $56.5 \%$ & 10 & $41.7 \%$ \\
\hline Total & 208 & $100.0 \%$ & 76 & $100.0 \%$ & 108 & $100.0 \%$ & 24 & $100.0 \%$ \\
\hline \multicolumn{9}{|l|}{ Age } \\
\hline 19 & 1 & $0.5 \%$ & 0 & $0.0 \%$ & 1 & $0.9 \%$ & 0 & $0.0 \%$ \\
\hline 2029 & 32 & $15.4 \%$ & 6 & $7.9 \%$ & 22 & $20.4 \%$ & 4 & $16.7 \%$ \\
\hline 3039 & 37 & $17.8 \%$ & 5 & $6.6 \%$ & 24 & $22.2 \%$ & 8 & $33.3 \%$ \\
\hline 4049 & 36 & $17.3 \%$ & 10 & $13.2 \%$ & 22 & $20.4 \%$ & 4 & $16.7 \%$ \\
\hline 5059 & 42 & $20.2 \%$ & 18 & $23.7 \%$ & 19 & $17.6 \%$ & 5 & $20.8 \%$ \\
\hline 6069 & 43 & $20.7 \%$ & 24 & $31.6 \%$ & 16 & $14.8 \%$ & 3 & $12.5 \%$ \\
\hline 7079 & 15 & $7.2 \%$ & 12 & $15.8 \%$ & 3 & $2.8 \%$ & 0 & $0.0 \%$ \\
\hline 80 & 2 & $1.0 \%$ & 1 & $1.3 \%$ & 1 & $0.9 \%$ & 0 & $0.0 \%$ \\
\hline Total & 208 & $100.0 \%$ & 76 & $100.0 \%$ & 108 & $100.0 \%$ & 24 & $100.0 \%$ \\
\hline \multicolumn{9}{|l|}{ Education } \\
\hline Basic & 20 & $9.6 \%$ & 12 & $15.8 \%$ & 8 & $7.4 \%$ & 0 & $0.0 \%$ \\
\hline High school & 74 & $35.6 \%$ & 30 & $39.5 \%$ & 38 & $35.2 \%$ & 6 & $25.0 \%$ \\
\hline College & 49 & $23.6 \%$ & 15 & $19.7 \%$ & 27 & $25.0 \%$ & 7 & $29.2 \%$ \\
\hline University & 61 & $29.3 \%$ & 18 & $23.7 \%$ & 34 & $31.5 \%$ & 9 & $37.5 \%$ \\
\hline Others & 4 & $1.9 \%$ & 1 & $1.3 \%$ & 1 & $0.9 \%$ & 2 & $8.3 \%$ \\
\hline Total & 208 & $100.0 \%$ & 76 & $100.0 \%$ & 108 & $100.0 \%$ & 24 & $100.0 \%$ \\
\hline
\end{tabular}

\subsection{Attitude toward Edible Wild Plants}

3.2.1. Preferences of Edible Wild Plants. In the second part of the questionnaire sheet, the interviewees were asked how frequently they purchase, eat, and collect edible wild plants during the harvest season of edible wild plants from January to June. The results were listed in Table 4. From Table 4, we can see that more than $40 \%$ of the respondents from the three groups take sansei more than once a week.

Respondents from group I not only eat and harvest edible wild plants, they also purchased more often than the other two groups. The respondents of group I from Noto Region use and harvest wild edible plants much more often than group II and group III. A big difference was in the frequency of taking and collecting edible plant. Around 78\% of the respondents from Group I take sansai more than once a week, comparing to only $42 \%$ of respondents from Group II. Around $48 \%$ of respondents from group I collect sansai more than once a week, comparing to only $12.9 \%$ of respondents from group II. Around $87 \%$ of respondents from group II answered that they have only a few or no experience of sansai harvesting. Respondents from group I buy sansai slightly more often than those from group II.

Difference was also found in the results of where to buy sansai (Table 6). About 61 respondents from group I answered this question, among which $62.3 \%$ usually buy from a famers' market, and $34.4 \%$ usually buy from a supermarket. Among the total 77 respondents from group II, around $28.6 \%$ of which usually buy from a farmers' market, and around two-thirds of them usually buy from a supermarket.

Respondents were also asked to select the edible wild plant species of three sources; buying, harvesting, and receiving form their relatives. In this question (multiple choice question), around 12 kinds of wild plants, which are commonly used in Ishikawa Prefecture, were listed: Petasites japonicus, Allium schoenoprasum, Aralia elata, Aralia cordata, Allium victorialis var. platyphyllum, Matteuccia struthiopteris, Pteridium aquilinum var. latiusculum, Petasites japonicus, Elatostema umbellatum var. majus, Oenanthe javanica, Osmunda japonica, and bamboo shoot sprout. The result of this question is closely correlated to that of the previous question of frequency. The result shows that more than one-fourth of the respondents harvest 7 kinds of Petasites japonicus and its bud, Aralia elata, Aralia cordata, Matteuccia struthiopteris, Pteridium aquilinum var. latiusculum, and Elatostema umbellatum var. majus. More than one-fourth of the respondents from group I buy Aralia cordata, Pteridium aquilinum, and Petasites japonicus; more than one-fourth of the respondents from groups II and III buy Aralia elata and Pteridium aquilinum var. latiusculum. The result of this question reveals that Aralia elata, Aralia cordata, and Pteridium aquilinum var. latiusculum are among the best popular edible wild plants. 
TABLE 4: Frequency of purchase, eating, and harvest edible wild plants.

\begin{tabular}{|c|c|c|c|c|c|c|}
\hline \multirow{2}{*}{ Frequency } & \multicolumn{2}{|c|}{ Group I } & \multicolumn{2}{|c|}{ Group II } & \multicolumn{2}{|c|}{ Group III } \\
\hline & Number & Percentage & Number & Percentage & Number & Percentage \\
\hline \multicolumn{7}{|l|}{ Frequency of purchase } \\
\hline$>3$ times/week & 8 & $10.5 \%$ & 3 & $2.8 \%$ & 1 & $4.2 \%$ \\
\hline 1-3 times/week & 21 & $27.6 \%$ & 21 & $19.4 \%$ & 5 & $20.8 \%$ \\
\hline Several times in a year & 22 & $28.9 \%$ & 44 & $40.7 \%$ & 12 & $50.0 \%$ \\
\hline Never & 25 & $32.9 \%$ & 40 & $37.0 \%$ & 6 & $25.0 \%$ \\
\hline No response & 0 & & 0 & & 0 & \\
\hline Total & 76 & $108.0 \%$ & 108 & $24.0 \%$ & 24 & \\
\hline \multicolumn{7}{|l|}{ Frequency of eating } \\
\hline$>3$ times/week & 28 & $36.8 \%$ & 11 & $10.2 \%$ & 4 & $16.7 \%$ \\
\hline 1-3 times/week & 31 & $40.8 \%$ & 34 & $31.5 \%$ & 6 & $25.0 \%$ \\
\hline Several times in a year & 16 & $21.1 \%$ & 59 & $54.6 \%$ & 13 & $54.2 \%$ \\
\hline Never & 1 & $1.3 \%$ & 4 & $3.7 \%$ & 1 & $4.2 \%$ \\
\hline No response & 0 & & 0 & & 0 & \\
\hline \multicolumn{7}{|l|}{ Frequency of harvest } \\
\hline$>3$ times/week & 15 & $19.7 \%$ & 4 & $3.7 \%$ & 0 & $0.0 \%$ \\
\hline 1-3 times/week & 21 & $27.6 \%$ & 10 & $9.3 \%$ & 2 & $8.3 \%$ \\
\hline Several times in a year & 26 & $34.2 \%$ & 35 & $32.4 \%$ & 7 & $29.2 \%$ \\
\hline Never & 13 & $17.1 \%$ & 59 & $54.6 \%$ & 15 & $62.5 \%$ \\
\hline No response & 1 & $1.3 \%$ & 0 & $0.0 \%$ & 0 & $0.0 \%$ \\
\hline Total & 76 & $108.0 \%$ & 108 & $24.0 \%$ & 24 & $100.0 \%$ \\
\hline
\end{tabular}

3.2.2. Binary Logistic Model: Effects of Age, Education, and City of Residence on Behavior of Purchasing, Collecting, and Eating Edible Wild Plants. We use the binary logistic regression (BLR) to identify relationship of frequency of purchasing, eating, and harvesting and the characterize of respondents. The general form of binary logistic regression is as follows:

$$
\operatorname{Probability}(y)=\frac{1}{1+\left(\operatorname{Exp}^{-\left(b_{0}+b_{1} x_{1}+b_{2} x_{2}+b_{3} x_{3}+\cdots+b_{n} x_{n}\right)}\right)} \text {. }
$$

Three models are used, where $y 1, y 2$, and $y 3$ are dependent variables and all are binary response variables $(0$ or 1). $y 1=1$ means the higher frequency of purchasing (more than 1 time one week), $y 1=0$ means others; $y 2=1$ means the higher frequency of eating (more than 1 time one week), $y 2=0$ means others; $y 3=1$ means the higher frequency of harvesting (more than 1 time one week), $y 3=0$ means others. Age, gender, education, the birthplace of the respondents, and the survey site are included in the models.

The results of the regression are showed in Table 5. Our two models based on binary logistic regression showed significant influence of respondents' demographic characteristics on their behaviors of purchase, harvesting, or eating edible wild plants (Table 5). Preference differences were found in the respondents with difference in residence of city, age, and education. Respondents from Noto Peninsula had a higher frequency of harvesting and eating edible wild plants than those from Kanazawa City and the other areas. Younger respondents, whose ages were less than 39, were much less involved with edible wild plants. The young group ate, harvested, or bought at a lower frequency than the group older than 40 yrs. Middle-aged group who were in their $40 \mathrm{~s}$ and 50s harvested less than the respondents older than 60 . Respondents with higher education harvested edible wild plants less than those whose education was basic education or lower than basic education.

3.2.3. Attitude toward Edible Wild Plants. The result of mean points of a Likert scale was calculated as Table 7. In this section of the questionnaire, the respondents were asked to check one choice with a number from 1 to 5 , which could best represent their attitudes towards the statement. Compare the results of group I and group II, it was found that the respondents from the two groups had little difference of the opinions on the five statements among the total 9 statements. Both the groups strongly agree or agree that sansai is delicious, healthy, and safe food, are one of representative traditional food culture, and environmental friendly without input of chemical fertilizers. Concerning the statements of being cheap, easy to cook, and easy to buy, group I held a more positive attitude toward them than group II.

3.2.4. Respondents' Concern towards Edible Wild Plants. In the last part of the questionnaire sheet, respondents were encouraged to write down any comments related to the harvest, consumption of wild edible plants. We got a total number of 64 results: among them 21 were from group I of Noto Region, 34 from group II, and 9 from group III. The results of this part were summarized in Table 8 . After reading all data, data of open-ended question were categorized into 
TABle 5: Binary logistic model: effects of age, education, and city of residence on behavior of purchasing, collecting, and eating edible wild plants.

\begin{tabular}{|c|c|c|c|}
\hline Parameter (std.error) & $\begin{array}{c}\text { Model } 1 \\
\text { Model } 1 \text { (frequency of purchase) }\end{array}$ & $\begin{array}{c}\text { Model } 2 \\
\text { Model } 2 \text { (frequency of eating) }\end{array}$ & $\begin{array}{c}\text { Model } 3 \\
\text { Model } 3 \text { (frequency of harvest) }\end{array}$ \\
\hline Survey site & $0.6033(0.4353)$ & $0.5379(0.4067)$ & $0.6798(0.5234)$ \\
\hline Gender & $-0.1663(0.3303)$ & $0.4281(0.3307)$ & $0.4883(0.3844)$ \\
\hline Residence & $0.0438(0.4347)$ & $0.9926^{* *}(0.4517)$ & $1.2659 * *(0.4935)$ \\
\hline Age (20-39) & $-1.2682^{* *}(0.5270)$ & $-1.8635^{* * *}(0.5015)$ & $-1.3372 * *(0.5510)$ \\
\hline Age (40-59) & $0.1085(0.4082)$ & $-0.7708(0.4709)$ & $-1.0882 * *(0.4640)$ \\
\hline Education ( basic) & $-0.0406(0.5870)$ & $-0.4123(0.6900)$ & $-1.2198^{* *}(0.6200)$ \\
\hline Education (high school) & $0.2133(0.5829)$ & $0.2898(0.7101)$ & $-0.0713(0.6098)$ \\
\hline Constant & $-1.0360 *(0.5864)$ & $0.3330(0.6541)$ & $-1.2007 *(0.6246)$ \\
\hline-2 log likelihood & 226.2082 & 231.4197 & 180.0617 \\
\hline Nagelkerke R square & 0.1322 & 0.3090 & 0.3379 \\
\hline
\end{tabular}

*** Significant at 0.01 level.

** Significant at 0.05 level.

* Significant at 0.10 level.

TABLE 6: Where do you buy the edible wild plants from?

\begin{tabular}{|c|c|c|c|c|c|c|c|c|}
\hline & \multicolumn{2}{|c|}{ Total } & \multicolumn{2}{|c|}{ Noto Peninsula } & \multicolumn{2}{|c|}{ Ishikawa Prefecture except Noto Peninsula } & \multicolumn{2}{|c|}{ Outside Ishikawa Prefecture } \\
\hline & Number & Percentage & Number & Percentage & Number & Percentage & Number & Percentage \\
\hline $\begin{array}{l}\text { Farmers' } \\
\text { market }\end{array}$ & 68 & 0 & 38 & 1 & 22 & 0 & 8 & 0 \\
\hline Supermarket & 83 & 1 & 21 & 0 & 52 & 1 & 10 & 1 \\
\hline Others & 7 & 0 & 4 & 0 & 3 & 0 & 0 & 0 \\
\hline No answer & 1 & 0 & 0 & 0 & 0 & 0 & 1 & 0 \\
\hline Total & 157 & 1 & 61 & 1 & 77 & 1 & 19 & 1 \\
\hline
\end{tabular}

TABLE 7: Respondents' attitude toward edible wild plants.

\begin{tabular}{lccc}
\hline & Group I & Group II & Group III \\
\hline Delicious & 4.6 & 4.5 & 4.6 \\
Healthy & 4.5 & 4.3 & 4.6 \\
Cheap & 3.8 & 3.1 & 3.6 \\
Easy to cook & 3.9 & 3.1 & 3.3 \\
Availability & 4.4 & 3.6 & 3.7 \\
Safe food & 4.6 & 4.0 & 4.3 \\
Representing traditional food & 4.5 & 4.1 & 4.3 \\
culture & 4.5 & 4.1 & 4.3 \\
Environmental friendly &
\end{tabular}

Note: the numbers of mean attitudes of each group were calculated based on the 5-point Likert scale. A point of 5 refers to strongly agree; 1 point refers to strongly disagree.

three groups: consumption, harvesting, and others. This part is strongly relevant to the third part of the questionnaire of respondents' attitude towards wild edible plants.

Regarding the availability, respondents from group I were satisfied of being accessible to edible wild plants in the forest and in the market. They expressed their contentment of being at the production site and harvesting the fresh wild edible plants of rich species. In contrast, respondents from both group II and III expressed difficulty in availability of wild edible plants.

Consistent to the above part of questionnaire survey results, respondents emphasized their liking of wild edible plants of being delicious, safe, and healthy. However, about 4 respondents from group II mentioned the difficulty of cooking.

Regarding the harvest of wild edible plants, three of the respondents complained about the harvest by outsiders. Six respondents concerned about the sustainable harvest of wild edible plants. The respondents from group II expressed their desire to be accessible to the forested mountain and fresh wild edible plants.

3.3. Commercial Harvests and the Attitudes of Forest Owners towards Edible Wild Plants Harvest. Based on the results of questionnaire survey, in-depth interviews were conducted to clarify the attitudes of forest owners and forest managers. Commercial harvesters who might also collect wild edible plants from others' private forest were reluctant to cooperate with the survey. There is a difficulty of collecting the data of the residents involved with commercial harvest. Japan Agricultural Cooperatives (JA) in Noto Region launched a project to market edible wild plants to the central market in Kanazawa, the capital city of Ishikawa Prefecture. According 
TABLE 8: Summary of respondents' concern towards consumption and harvest of edible wild plants.

\begin{tabular}{|c|c|c|c|c|c|c|}
\hline \multirow[b]{2}{*}{ Categories } & \multicolumn{2}{|l|}{ Group I } & \multicolumn{2}{|l|}{ Group II } & \multicolumn{2}{|c|}{ Group III } \\
\hline & Subcategories & Number & Subcategories & Number & Subcategories & Number \\
\hline \multirow{6}{*}{ Consumption/consumers } & Easy to buy & 2 & Difficulty in availability & 8 & $\begin{array}{l}\text { Difficulty in } \\
\text { availability }\end{array}$ & 2 \\
\hline & $\begin{array}{l}\text { Delicious and } \\
\text { fresh to be in the } \\
\text { production sites }\end{array}$ & 7 & $\begin{array}{l}\text { Somewhat prepared or cooked } \\
\text { for sales due to the difficulty of } \\
\text { cooking }\end{array}$ & 4 & $\begin{array}{l}\text { We like wild } \\
\text { edible plants for } \\
\text { they are natural } \\
\text { food }\end{array}$ & 5 \\
\hline & $\begin{array}{l}\text { Sometimes } \\
\text { expensive }\end{array}$ & 1 & $\begin{array}{l}\text { Receive a lot from relatives or } \\
\text { friends }\end{array}$ & 4 & & \\
\hline & Safe food & 1 & $\begin{array}{l}\text { We like wild edible plants and } \\
\text { hope to maintain the production }\end{array}$ & 3 & & \\
\hline & & & Do not eat & 2 & & \\
\hline & & & Variety became less & 1 & $\begin{array}{l}\text { Receive a lot from } \\
\text { relatives or } \\
\text { friends }\end{array}$ & 4 \\
\hline \multirow{4}{*}{ Harvest / collectors } & Enjoy collecting & 1 & $\begin{array}{l}\text { Wish to harvest and cook by } \\
\text { himself, however, lacks } \\
\text { information of growth of wild } \\
\text { edible plant }\end{array}$ & 2 & $\begin{array}{l}\text { Construction of } \\
\text { golf courses and } \\
\text { houses should be } \\
\text { limited in the } \\
\text { forested } \\
\text { mountains }\end{array}$ & 1 \\
\hline & $\begin{array}{l}\text { The number is } \\
\text { decreasing with a } \\
\text { lot of outsiders }\end{array}$ & 4 & $\begin{array}{l}\text { Access to the mountain should } \\
\text { be legally supported }\end{array}$ & 3 & $\begin{array}{l}\text { Properly manage } \\
\text { the forest in order } \\
\text { to get access to it }\end{array}$ & 1 \\
\hline & $\begin{array}{l}\text { Should be easier } \\
\text { to sell harvested } \\
\text { wild edible plant }\end{array}$ & 3 & $\begin{array}{l}\text { Should be collected in a } \\
\text { sustainable way }\end{array}$ & 6 & & \\
\hline & $\begin{array}{l}\text { Food bank for } \\
\text { emergency, for } \\
\text { example, } \\
\text { starvation or wars }\end{array}$ & 1 & $\begin{array}{l}\text { Should be revaluated as part of } \\
\text { traditional culture and passed } \\
\text { down to next generation }\end{array}$ & 1 & & \\
\hline \multirow[t]{2}{*}{ Others } & $\begin{array}{l}\text { Use abandoned } \\
\text { field to cultivate } \\
\text { wild edible plant }\end{array}$ & 1 & & & & \\
\hline & & 21 & & 34 & & 9 \\
\hline
\end{tabular}

to the data provided by Anamizu Town Office, JA helped the harvesters from Anamizu Town to sell around 11 species of edible wild plants since 2008. The total sales ranged from JPY 2 million to 8 million (see Table 9) during the past three years.

Noto Forestry Cooperative (FCA), which is an organization of forest owners in charge of forest management in Oku Noto at the north part of Noto Peninsula, was interviewed to explore into forest owners' attitudes toward edible wild plants collecting by recreational visitors and commercial harvesters. Head and counselor of FCA were interviewed, respectively. Three forest owners, Mr. and Mrs. Sakamoto and Mr. Kobayashi, were interviewed. Problems listed by FCA and interviewed forest owners include several aspects. First, garbage disposal was listed as the major factor. Secondly, some forest owners partly rely on the edible wild plants for livelihood. The recreational visitors and outside commercial harvesters threaten the benefits of this group. In particular, the prices of edible fungi of high value, for example, matsutake, have multiplied with the increasing demand of fungi in the market. Thirdly, the improper ways of collecting are concerned. Both forest owners and local harvesters mentioned that it will hurt the regeneration in the next year if the roots of wild plants were hurt or taken. Osmunda japonica, a dioecious species, is also a poplar fern sprout. There exists a tradition in Noto Region that only new sprouts of female $O$. japonica are harvested, while male O. japonica are left. The new sprouts of some tree species are also edible. For some species such as Aralia elata and Acanthopanax sciadophylloides are not allowed to pick all new leaves, otherwise, the bare tree would wither.

In the Noto Region, about $70 \%$ of the land is covered with forests, among which private forest consists of $87 \%$ of the total forest area (Table 10). Our interview with the owners of the private forest found that they generally accept that their friends or residents from the same community to collect wild edible plants from their forested mountain. At the same time, the majority of them are strongly against the 
TABLE 9: Forest area by ownership in the Noto Region.

\begin{tabular}{|c|c|c|c|c|c|c|c|}
\hline \multicolumn{8}{|c|}{ Forest area (ha) } \\
\hline & $\begin{array}{l}\text { Land area } \\
\text { (ha) }\end{array}$ & Total & National forest & $\begin{array}{c}\text { Public forest owned by } \\
\text { local authorities and } \\
\text { corporate* }^{*}\end{array}$ & Private forest & $1^{* *}$ & $2^{* * *}$ \\
\hline Nanao City & 31796 & 20433 & 53 & 1702 & 18678 & $91.4 \%$ & $64.3 \%$ \\
\hline Wajima City & 42625 & 32735 & 47 & 5759 & 26929 & $82.3 \%$ & $76.8 \%$ \\
\hline Suzu City & 24720 & 18699 & 43 & 3541 & 15115 & $80.8 \%$ & $75.6 \%$ \\
\hline Hakui City & 8196 & 2919 & 4 & 480 & 2435 & $83.4 \%$ & $35.6 \%$ \\
\hline Shika Town & 24655 & 16225 & 69 & 1846 & 14310 & $88.2 \%$ & $65.8 \%$ \\
\hline $\begin{array}{l}\text { Nakanoto } \\
\text { Town }\end{array}$ & 8936 & 5113 & 5 & 789 & 4319 & $84.5 \%$ & $57.2 \%$ \\
\hline $\begin{array}{l}\text { Anamizu } \\
\text { Town }\end{array}$ & 18324 & 13548 & 30 & 1401 & 12117 & $89.4 \%$ & $73.9 \%$ \\
\hline Noto Town & 27346 & 20773 & 57 & 2775 & 17941 & $86.4 \%$ & $76.0 \%$ \\
\hline Total & 186598 & 130445 & 308 & 18293 & 111844 & $85.7 \%$ & $69.9 \%$ \\
\hline
\end{tabular}

Note: data in this table was calculated based on Annual Report of statistics of Agriculture, Forestry, and Fisheries in Ishikawa Prefecture (year 2007-2008).

* The number is the combination of public forest owned by local authorities and Green Resource Public Corporation.

$1^{* *}$ refers to the percentage of private forest to the total forest area.

$2 * *$ refers to the percentage of forested area to the total land area.

TABLE 10: The total amount and sales of edible wild plants sold by JA to the central market, Kanazawa, of Ishikawa Prefecture.

\begin{tabular}{lcc}
\hline & Amount $(\mathrm{kg})$ & Sales (JPY) \\
\hline 2008 & $1,332.15$ & $2,060,288$ \\
2009 & $9,953.45$ & $8,149,535$ \\
2010 & $5,209.45$ & $3,287,458$ \\
\hline
\end{tabular}

Data source: the number is provided by Anamizu Town Office.

intrusion by outsiders from other community, in particular from other prefecture, including the forest recreationists from the urban area. In order to prevent outsiders from entering the forests, access to the forests is prohibited. According to Counselor of Noto FCA, Mr. Yosaburou Yachi, around $60 \%$ of the forest was set up with a keep-out sign. Harvesters outside of the community and commercial harvesters are in general prohibited to enter the forest.

\section{Discussion}

4.1. Edible Wild Plants as Traditional Food Culture. It was found that there exist abundant edible wild plants in Noto Region, in consistency with some previous studies in other parts of Japan [13-15]. About 200 species can be used as food or medicine, the majority of which were in the forest. Noto Region is a treasure box full of natural and healthy food. A total amount of edible wild plants was estimated to be around 200 species in other parts of Japan. Only about $15 \%$ of them were eaten by local residents $[17,18]$.

This study revealed that people living in Noto Region, as well as those in nearby urban areas have very close connection to edible wild plants as that a majority of the respondents have eaten, bought, and harvested them very often during the harvest season of edible wild plants.
Respondents surveyed had a positive attitude towards edible wild plants. Edible wild plants were labeled by a majority of respondents from remote mountainous areas as well as from urban areas as being tasty, healthy, and safe food. Edible wild plants are considered to be representative traditional food related to local culture.

Some early researches [13] also reported that the people of Japan have considered edible wild plants as tasty food [14, 15]. It was also reported that Japanese consumers considered edible wild plants as expensive, while, this survey found that consumers in Noto Region considered the prices as reasonable. Comparing with reports in other areas or in previous year, respondents in this survey had a more positive assessment of edible wild plants.

Survey also found Farmers' Market as an important marketing channel of wild edible plants, in particular in Noto Peninsula. In contrast, around two-thirds of consumers in metropolitan city bought from a supermarket or a vegetable store [16].

With the ageing and depopulation in rural areas, the traditional knowledge related to edible wild plants is facing the threat of degradation. The loss of traditional ecological knowledge associated with wild plants negatively affects the lives and health of traditionally living rural communities [19]. Although numerous previous studies report that traditional knowledge related to wild edible plants has greatly declined in Africa [19] and Japan [4], due to the impacts of the drivers of ageing and migration. Findings in this study revealed that residents from not only the mountainous areas and urban areas hold very positive attitude towards edible plants and their lives are closely related to wild plants. A majority of respondents agreed that edible wild plants are important part of their traditional diet. Traditional knowledge related to eating and harvesting edible wild plants are still passed on, while at some extent declining. 
How to maintain and dissimilate the traditional knowledge related to wild edible plants must be considered as part of revitalization of rural area in Japan. Young generations must be the foci of education of traditional ecological knowledge. Urban residents from the nearby cities should be included as potential recreationists from the aspects of dynamically conserving traditional culture, in particular endemic dietary and revitalizing rural areas by enhancing the attractions of forest and nature to increase visitors to remote areas.

\subsection{Challenges to Develop the Recreational Uses of Edible Wild} Plants. Abundant species of edible wild plants and being able to access low hills (satoyama) without wild boars or bears in Noto Peninsula have attracted not only local residents but also urban residents from Ishikawa Prefecture and even other prefectures to harvest sansai. It was found in this study that private forest owners do not consider these outsiders desirable.

Many studies [20] found the importance of continued public ownership and of continued (and enhanced) multiuse management strategies. Thus, we assume that private ownership of forest resources is a major impediment to recreational use of nature resource. In Japan, private forests hardly participate in providing recreational activities, and researches related to recreational activities on private land or by privately funds are very rare [21].

Recreational use of forests must be reevaluated since forests are considered to be multifunctional. Forest and Forestry Basic Act was radically revised based on Basic Forestry Law (1964) and enacted in 2001. It designates the basic principles for forest policies in the 21st century. In this act, it places the sustainable development of multifunctions of the forest as the basic ideology. Among the multi-functions of the forest mentioned in this new Forest and Forestry Basic Act, recreational function of the forest as to enhance public health was evaluated the same as other forest products.

Maintaining NTFP resources was less critical for the subsistence needs of the majority of the public and therefore did not rank high as a management priority. The emphasis may have shifted from subsistence to commercial and recreational pursuits, harvesting [22]. Commercial harvesting and marketing of edible wild plants in Noto Region are still far from being successful given the small amount and unsteady growth of the total amount and price (Table 8).

\section{Conclusion}

With the rapid urbanization, increase of abandoned farmlands and undermanaged forests in the past decades, the changing rural landscape has posed threats towards the loss of biodiversity and associated environmental services. It is evident that interest has been given to biomass production and restoration of ancient woods in the coppice woodlands, while little attention so far has been paid to the social dimension. There is a danger of neglecting the potential for coppices as socially important woodland types. Traditional knowledge has been emphasized as the most promising cognitive response to the balance of protection of satoyama landscape and other traditionally managed forest [23] and sustainable use of natural resources. A FAO's Globally Important Agricultural Heritage System (GIAHS) pilot site in the Noto Peninsula was designated to foster dynamic conservation and sustainable use of local natural resources.

Our survey in Ishikawa Prefecture found that there exist abundant species of edible wild plants in satoyama, and local people not only from remote areas but also from urban areas have highly positive attitude towards edible wild plants. Developing the sustainable use of natural resources in satoyama by promoting the harvest and consumption of edible wild plants could be considered as an important means of rural vitalization. It was found that farmers' market of Japan Agricultural Cooperatives (JA) has played a vital role in marketing edible wild plants. Young respondents had a low frequency of consuming edible wild plants. One of our findings is consistent with previous studies $[17,18]$ that it is an urgent issue to educate young people with traditional knowledge in order to prevent from the loss of relevant traditional culture.

NTFP extraction does not have to be seen as a threat to biodiversity, but rather can be seen as a tool for creating socioeconomic relationships that are dependent on healthy, biodiverse ecosystems. It has been argued that anthropogenic disturbance of intermediate impact has developed rich biodiversity in the secondary forest [24]. Some local residents considered that edible wild plants collectors from other communities including urban residents will threaten their interest.

An early report recommended having forest recreationists pay a right of access to harvesting wild edible plants [25]. Japan Satoyama Satoumi Assessment [2] recommends the idea of the "new commons" to create a new management system by local government and NPOs and NGOs [2]. In particular, we argue that "new commons" should include urban residents to access the forests for nontimber forest product, for example, edible wild plants. In Nordic European countries, there is the traditional right of "Everyman's right," which includes berry picking, mushroom collecting, and free access for all recreation and sports activities [26]. It is obvious that, in Japan, the customary law with limiting the access of residents from other communities, and the current private ownership have excluded the urban residents to access the forested hills. It is suggested that those private forests which were abandoned in terms of management in remote region could be accessible to urban residents for obtaining wellbeing of forest resources through the administrative supports at some extent. JA and Forestry Cooperatives (FCA) should play an important role in achieving the GIAHS goal of dynamic conservation and sustainable management in the Noto Peninsula.

\section{Acknowledgments}

The authors wish to thank Ms. Fujiko Taniguchi and numerous local people for their sincere cooperation during the field surveys and for providing local knowledge for this research. Thanks also go to Ms. Anne McDonald and 
Dr. Raquel Moreno-Penaranda for their invaluable comments. The authors owe sincere thanks to Ms. Atsuko Hasegawa for her help with proofreading the questionnaire sheet in Japanese. Thanks also go to the Editor-in-Chief and the two anonymous reviewers for their invaluable comments.

\section{References}

[1] K. Takeuchi, "The nature of satoyama landscape," in Satoyama the Traditional Rural Landscape of Japan, K. Kakeuchi, R. D. Brown, I. Washitani, A. Tsunekawa, and M. Yokohari, Eds., Springer, Tokyo, Japan, 2001.

[2] A. K. Duraiappah, K. Nakamura, K. Takeuchi, and M. Nishi, "Satoyama-Satoumi Ecosystems and Human Well-Being: Assessing Trends to Rethink a Sustainable Future," Policy Brief, vol. 2010, no. 7, 2010.

[3] Japan, Ministry of Agriculture, Forestry and Fisheries, Forestry Agency, Forestry Management Improvement Division, Special Forest Products Office For promotion edible wild plant culture and industry to revitalize mountainous villages. Sanrin, 1450: 40-45, 2005 (in Japanese).

[4] G. Cetinkaya, "Challenges for the maintenance of traditional knowledge in the Satoyama and Satoumi ecosystems, Noto Peninsula, Japan," Human Ecology Review, vol. 16, no. 1, pp. 27-40, 2009.

[5] United Nations University-Institute of Advanced Studies (UNU-IAS), “Japan's Satoyama Satoumi Assessmnt: Socialecological Productive Landscape in Japan, Experiences and Lessons from Hokushinetsu Cluster," United Nations University, Tokyo, Japan, 2010, (in Japanese).

[6] I. Washitani, "Traditional sustainable ecosystem "SATOYAMA" and biodiversity crisis in Japan: conservation ecological perspectives," Global Environmental Research, vol. 5, pp. 119133, 2001.

[7] H. Kobori, "Current trends in conservation education in Japan," Biological Conservation, vol. 142, no. 9, pp. 1950-1957, 2009.

[8] S. Komaki, Flora in Kaga and Noto (Kaga Noto Noto Shokubutsu $Z u f u$ ), Society of Flora in Kaga and Noto, Noto Province, Japan, 1987, (in Japanese).

[9] K. Imai and M. Imai, Best Guide to Wild Edible Plants (Yoku Wakaru Sansai Daizukan), Nagaoka Shoten, Tokyo, Japan, 2007, (in Japanese).

[10] M. Ōsuga, Dictionary of Trees and Herbs Used as Medicine (Yakuboku Yakus Jiten), Rekishi Shunju Publishing Co., Ltd., 1997, (in Japanese).

[11] Food and Agriculture Organization of the United Nations (FAO), 2002, http://www.fao.org/nr/giahs/whataregiahs/definition/en/.

[12] Japan, Ministry of Agriculture, Forestry and Fisheries (MAFF), Statistics of Forestry Income in 2001, (in Japanese), 2003, http://www.maff.go.jp/j/tokei/kouhyou/ringyou_sansyutu/index.html.

[13] T. Sugiura and K. Kishimoto, "On the liking of wild vegetables and mushrooms by consumers," Journal of the Japanese Forestry Society, no. 1, pp. 31-38, 1989 (Japanese).

[14] M. Ono, Y. Shinoda, H. Minami, S. Jyo, T. Sato, and K. Kawakami, "Investigation on the state of utilization of wild plants in Hida District (Part I)," Journal of the Nagoya Women's College, vol. 14, pp. 31-45, 1968 (Japanese).

[15] M. Ono, Y. Shinoda, H. Minami, S. Jyo, T. Sato, and K. Kawakami, "Investigation on the state of utilization of wild plants in Hida District (Part II)," Journal of the Nagoya Women's College, vol. 15, pp. 59b-68b, 1968 (Japanese).

[16] T. Sugiura and H. Uchiyama, "The understanding of wild vegetables and mushrooms by supermarkets and superstores," Journal Agricultural Science Tokyo Nogyo Daigaku, vol. 42, no. 3, pp. 210-220, 1997.

[17] T. Sugiura, "Present situation of use of edible wild plants in our country and its possibilities in the future," Tokusan Joho, vol. 23, no. 8, pp. 22-25, 2002 (Japanese).

[18] T. Sugiura, "Present situation of use of edible wild plants in our country and its possibilities in the future," Tokusan Joho, vol. 28, no. 9, pp. 28-31, 2007 (Japanese).

[19] T. P. Dweba and M. A. Mearns, "Conserving indigenous knowledge as the key to the current and future use of traditional vegetables," International Journal of Information Management, vol. 31, no. 6, pp. 564-571, 2011.

[20] P. J. Smailes and D. L. Smith, "The growing recreational use of state forest lands in the Adelaide hills," Land Use Policy, vol. 18, no. 2, pp. 137-152, 2001.

[21] T. Ito, "A comparative review of forest recreation studies in Japan and the United States," Journal of the Japanese Forestry Society, vol. 85, no. 1, pp. 33-46, 2003 (Japanese).

[22] E. T. Jones and K. A. Lynch, "Nontimber forest products and biodiversity management in the Pacific Northwest," Forest Ecology and Management, vol. 246, no. 1, pp. 29-37, 2007.

[23] Y. W. Chun and K. I. Tak, "Songgye, a traditional knowledge system for sustainable forest management in Choson Dynasty of Korea," Forest Ecology and Management, vol. 257, no. 10, pp. 2022-2026, 2009.

[24] K. Katoh, S. Sakai, and T. Takahashi, "Factors maintaining species diversity in satoyama, a traditional agricultural landscape of Japan," Biological Conservation, vol. 142, no. 9, pp. 1930-1936, 2009.

[25] F. Ueno, "An essay on the native vegetables and the right of access in the mountain areas in relation to land resource management and recreational activity," Komazawa Geography, vol. 11, pp. 91-96, 1975.

[26] T. Sievänen, E. Pouta, and M. Neuvonen, "Participation in mushroom picking in Finland," in Social Roles of Forests for Urban Population, T. Ito and T. Nobuhiko, Eds., Japan Society of Forestry Planning Press; University of Tokyo, Tokyo, Japan, 2004. 

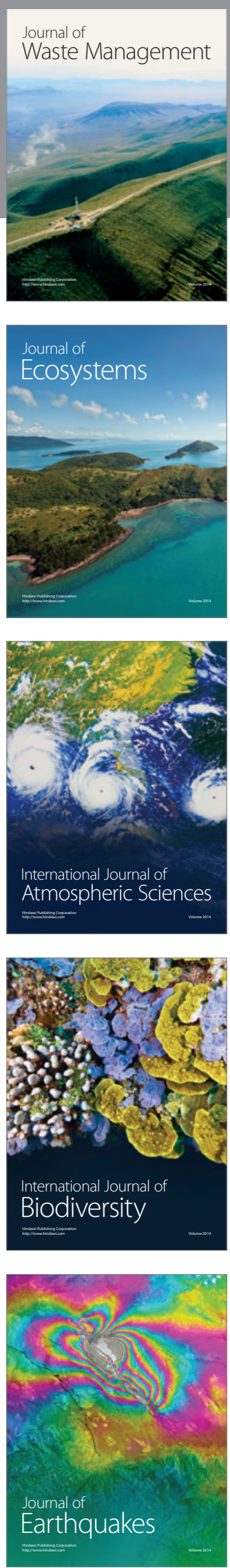
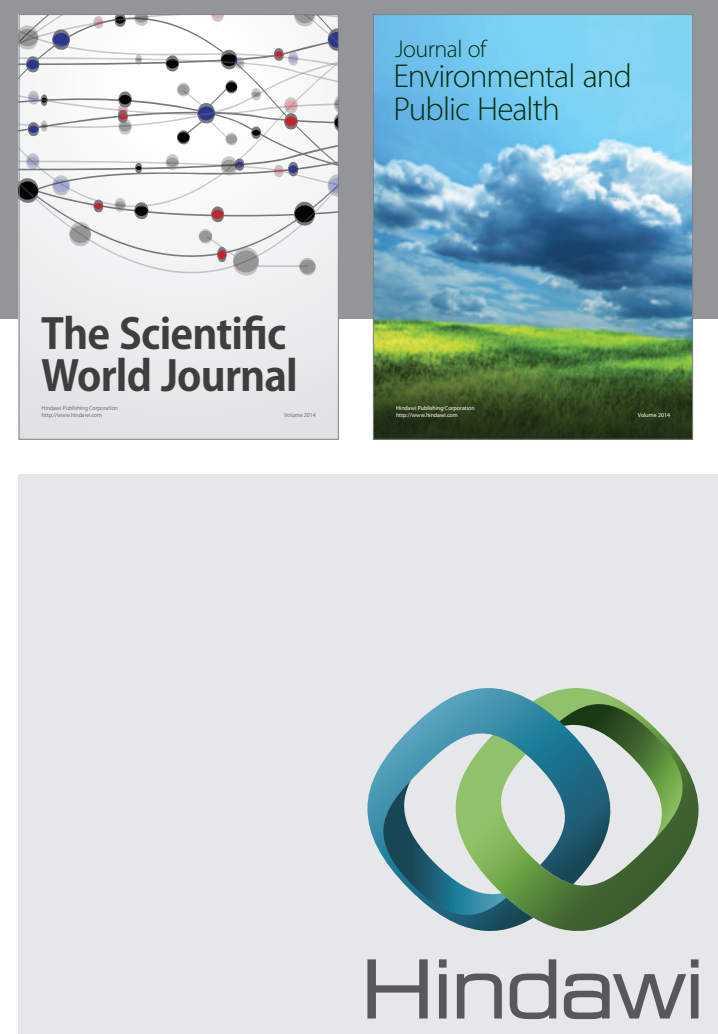

Submit your manuscripts at

http://www.hindawi.com
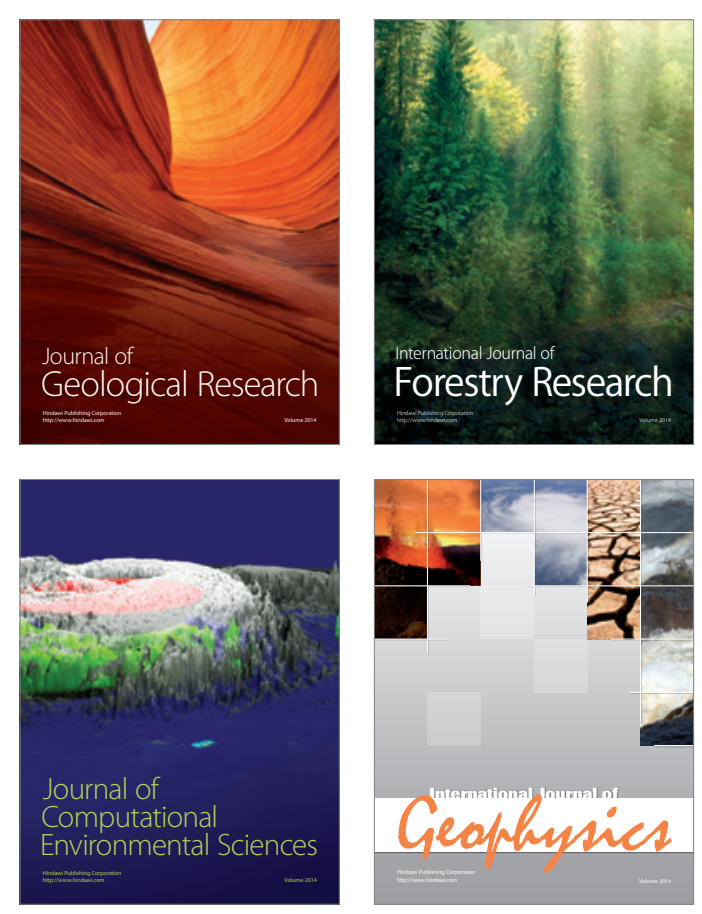
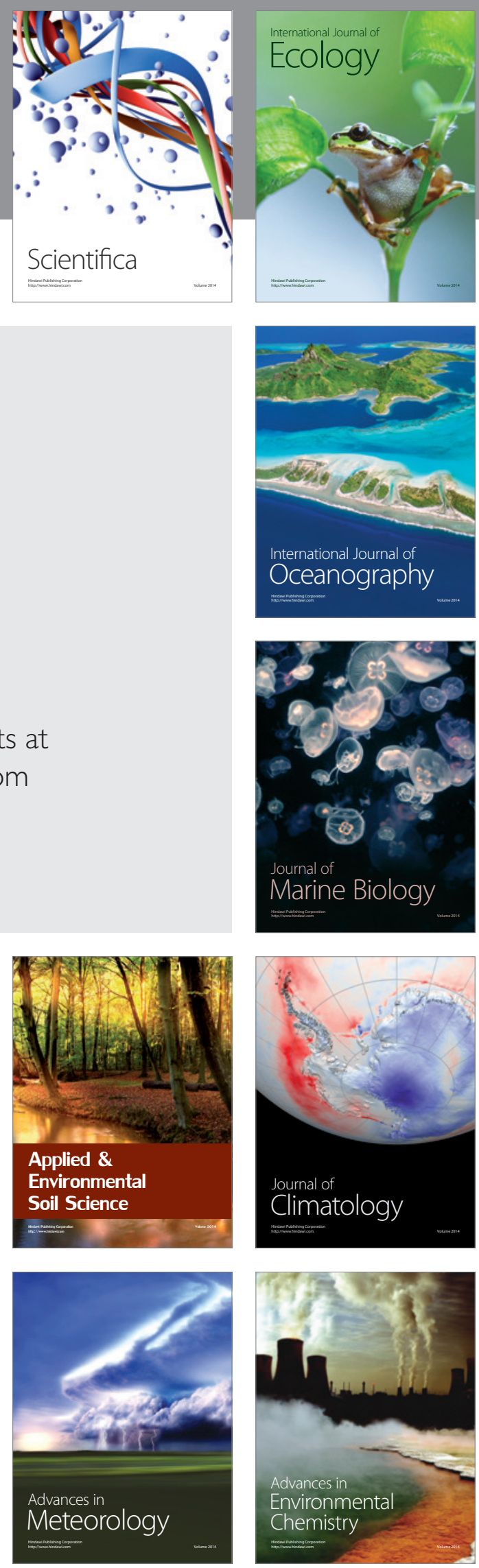